\title{
BMJ Open Protocol for the economic evaluation of a complex intervention to improve the mental health of maltreated infants and children in foster care in the UK (The BeST? services trial)
}

\author{
Manuela Deidda, ${ }^{1}$ Kathleen Anne Boyd,${ }^{1}$ Helen Minnis, ${ }^{2}$ Julia Donaldson, ${ }^{3}$ \\ Kevin Brown, ${ }^{4}$ Nicole R S Boyer, ${ }^{1}$ Emma Mclntosh, ${ }^{1}$ BeST study team
}

To cite: Deidda M, Boyd KA, Minnis $\mathrm{H}$, et al. Protocol for the economic evaluation of a complex intervention to improve the mental health of maltreated infants and children in foster care in the UK (The BeST? services trial). BMJ Open 2018;8:e020066. doi:10.1136/ bmjopen-2017-020066

- Prepublication history and additional material for this paper are available online. To view these files, please visit the journal online (http://dx.doi org/10.1136/bmjopen-2017020066).

Received 11 October 2017 Revised 19 January 2018 Accepted 26 January 2018

\section{Check for updates}

${ }^{1}$ Health Economics and Health Technology Assessment, University of Glasgow, Glasgow, UK

${ }^{2}$ Mental Health and Wellbeing, University of Glasgow, Glasgow, UK

${ }^{3}$ Glasgow Infant and Family Team, NSPCC, Glasgow, UK

${ }^{4}$ Family Assessment and Contact Service, Glasgow, UK

Correspondence to

Dr Manuela Deidda;

manuela.deidda@glasgow.ac.uk

\section{ABSTRACT}

Introduction Children who have experienced abuse and neglect are at increased risk of mental and physical health problems throughout life. This places an enormous burden on individuals, families and society in terms of health services, education, social care and judiciary sectors. Evidence suggests that early intervention can mitigate the negative consequences of child maltreatment, exerting long-term positive effects on the health of maltreated children entering foster care. However, evidence on costeffectiveness of such complex interventions is limited. This protocol describes the first economic evaluation of its kind in the UK.

Methods and analysis An economic evaluation alongside the Best Services Trial (BeST?) has been prospectively designed to identify, measure and value key resource and outcome impacts arising from the New Orleans intervention model (NIM) (an infant mental health service) compared with case management (CM) (enhanced social work services as usual). A within-trial economic evaluation and long-term model from a National Health Service/ Personal Social Service and a broader societal perspective will be undertaken alongside the National Institute for Health Research (NIHR)-Public Health Research Unit (PHRU)-funded randomised multicentre BeST?. BeST? aims to evaluate NIM compared with CM for maltreated children entering foster care in a UK context. Collection of Paediatric Quality of Life Inventory (PedsQL) and the recent mapping of PedsQL to EuroQol-5-Dimensions (EQ-5D) will facilitate the estimation of quality-adjusted life years specific to the infant population for a cost-utility analysis. Other effectiveness outcomes will be incorporated into a cost-effectiveness analysis (CEA) and cost-consequences analysis (CCA). A long-term economic model and multiple economic evaluation frameworks will provide decisionmakers with a comprehensive, multiperspective guide regarding cost-effectiveness of NIM. The long-term population health economic model will be developed to synthesise trial data with routine linked data and key government sector parameters informed by literature. Methods guidance for population health economic evaluation will be adopted (lifetime horizon, 1.5\% discount rate for costs and benefits, CCA framework, multisector perspective).
Strengths and limitations of this study

- Prospectively designed economic evaluation alongside complex intervention trial.

- First economic evaluation of a complex, public health intervention directed towards improving abused children's mental health in the UK setting.

- Preference-based (utility) outcome measures included to facilitate decision-making.

- Long-term economic model including linked data to other sectors, for example, crime/education.

- Challenges related to the complexity of this particular child services setting, with regard to the variability of the control arm (case management) in different sites across UK and to the different sources of data collection used.

Ethics and dissemination Ethics approval was obtained by the West of Scotland Ethics Committee. Results of the main trial and economic evaluation will be submitted for publication in a peer-reviewed journal as well as published in the peer-reviewed NIHR journals library (Public Health Research Programme).

Trial registration number NCT02653716; Pre-results.

\section{INTRODUCTION}

There is evidence that maltreated children are at greater risk for lifelong health and social problems, including mental illnesses, criminality, chronic diseases, disability ${ }^{1}$ and poorer quality of life. ${ }^{2}$ A history of child maltreatment is also associated with lower adult levels of economic well-being across a wide range of metrics, including higher levels of economic inactivity, lower occupational status, lower earnings and lower expected earnings. ${ }^{3}$ Existing research suggests a ripple effect caused by lower educational achievement, higher levels of truancy and expulsion reducing peak earning capacity by US $\$ 5000$ a 
year $^{4}$ or an average lifetime cost of US $\$ 210012$ per person ${ }^{1}$ when considering productivity losses and costs from healthcare, child welfare, criminal justice and special education.

Early interventions to promote the health and wellbeing of children have been shown to help mitigate the negative consequences of child maltreatment and have long-term positive effects on the health of maltreated children. ${ }^{5}$ Services are required that provide support to families as soon as they need it, and provide early permanency decisions. ${ }^{6}$ Interventions that exhibit these characteristics are most likely to improve children's mental health and well-being and reduce health and societal costs over the long term through increased likelihood that children will have higher educational achievements, successful lives and be less likely to be dependent on the state. In the short run, costs will be lowered by reducing social workers' time, avoiding several repeated decisions due to multiple placements. ${ }^{6}$

Several reports have highlighted the inadequacies of the UK's care system and the high costs associated with implementing new services ${ }^{6}$ as well as the increasing costs that are associated with cycling placements or returns to care. $^{7}$ Additionally, existing analyses have emphasised the challenges in conducting economic evaluations of interventions aimed at improving outcomes for maltreated children, which include the need for a long-term perspective, accounting for the context-specific nature of interventions, ${ }^{8}$ and overcoming obstacles of cross-comparison due to variations in methods, samples etc. ${ }^{9}$

\section{New Orleans intervention model for infant mental health in Glasgow and South London}

The New Orleans intervention model (NIM) (box 1) is based on the Tulane Infant Team programme ${ }^{10}$ and is being implemented in the UK through the collaboration between voluntary services National Society for the Prevention of Cruelty to Children (NSPCC), social (various City Councils including Glasgow and Croydon) and health services (including National Health Service (NHS) Greater Glasgow and Clyde and South London and Maudsley Trust) in the UK. A preliminary economic model exploring the likely costs and consequences of implementing the NIM in Glasgow concluded that NIM would be more costly, but the probability of repeated episodes is likely to fall significantly, as it involves both assessment and treatment phases as opposed to the assessment only nature of typical social services case management $(\mathrm{CM}){ }^{11}$

\section{The Best Services Trial}

The Best Services Trial (BeST?), led by the University of Glasgow, is a continuation and expansion of an internal pilot randomised controlled trial (RCT) that commenced December 2011 in Glasgow. During this preliminary study, approximately two-thirds of children coming into care in Glasgow were recruited and
Box 1 The New Orleans intervention model (NIM) intervention

NIM is an intervention which uses an infant mental health approach aiming to improve the quality of permanent placement decisions with the aim of improving outcomes for young children. The Tulane Infant Team, who developed NIM, assesses the mental health of every child under 5 years on reception into foster care and quality of the relationships between the child and their parents. A tailored intervention is then offered to each family aiming to improve parentchild relationships and child mental health. These assessments and the degree of change achieved through intervention inform recommendations to the legal system about the permanent future care of the child. Where change has been achieved which indicates it is safe, children are rehabilitated back to the birth family. If not, the recommendation is adoption or long-term care.

The NIM intervention will be delivered, in each site, by a multidisciplinary team comprising a child and adolescent psychiatrist, clinical psychologists, social workers therapists and administrative staff. Each member of clinical staff will receive specific training in assessment techniques and treatment delivery from the New Orleans and/or Glasgow team.

Participants randomised to NIM will be asked to take part in a detailed attachment-informed assessment involving each actual and potential caregiver. The assessment is manualised, standardised and uses structured interviews, self-report measures and observations. ${ }^{10}$ An intervention will then be tailored for every family, drawing on a small range of relationship-based therapeutic techniques all of which comply with the recommendations of a meta-analysis that examined ways of improving parental sensitivity. ${ }^{50}$ Parents will also be referred as required to other agencies for help with substance misuse, mental health issues or intrafamilial violence. The aim is to have the safest better outcome for the child, be this a recommendation of rehabilitation to birth family or adoption. ${ }^{51}$ Making well-informed permanent placement recommendations within 6-12 months could optimise physical, mental and social development, while also allowing parent the opportunity to make changes if possible.

randomised into receiving the NIM or enhanced CM. ${ }^{\mathrm{i}}$ The BeST? will continue as a definitive multicentre RCT expanding on the work currently taking place in Glasgow including an additional site in South London, England. Including a London site will increase generalisability of the findings which may have important policy implications for the UK. Details of the main trial study protocol are available elsewhere. ${ }^{12}$

NIM has demonstrated preliminary evidence of effectiveness in USA, ${ }^{10}$ but its effectiveness and cost-effectiveness in terms of improving the mental health of children coming into care following maltreatment is unknown in a UK setting. The absence of any standardised mental health services for maltreated infants in the UK which favours a 'social care'-oriented system, makes this an interesting case to analyse effectiveness

${ }^{\mathrm{i}}$ Enhanced case management is an enhanced service-as-usual in that a defined care pathway has been set and is being adhered to when a child comes into foster care. 
and cost-effectiveness of the NIM intervention. Initial US evidence suggests positive consequences of placement stability and improvements in infant mental health, as well as longer-term resulting benefits to academic performance, employability, and reduction in teenage delinquency and crime. ${ }^{11}$

\section{Aim}

The BeST? trial aims to evaluate the effectiveness and cost-effectiveness of NIM in improving the mental health of maltreated infants and children in foster care, the relationship between these children and their primary caregivers, and the timeliness of permanent placement decisions, compared with CM. The aim of the economic evaluation alongside the BeST? RCT is to establish the short-term and long-term cost-effectiveness of NIM compared with $\mathrm{CM}$ from both a health and societal perspective.

\section{METHODS AND ANALYSIS}

BeST? is a multisite RCT taking place in Glasgow and London, UK. These sites are characterised by a high level of deprivation and a large number of children coming to foster care, thus representing an interesting location to analyse effectiveness and cost-effectiveness of the NIM intervention.

Families who have a child entering care at either site, aged 0-60 months, will be randomised to receive NIM or CM. Data collection captures information regarding whoever the primary carer is at the time of follow-up (whether that be birth parent(s), grandparents, foster carers or adopted parents) and it will take place at baseline and at follow-up (15 months and 2.5 years). The primary outcome for the trial is the Strengths and Difficulties Questionnaire (SDQ) at 2.5 years: a sample size of 462 will have $90 \%$ power to detect an effect size of 0.35 , allowing for $25 \%$ loss to follow-up in this intention-totreat analysis. ${ }^{12}$

The economic evaluation will involve a within-trial economic analysis and a population health economic model considering the long-term impact of the NIM intervention on several government sectors where a mental health intervention of this kind is likely to have an impact.

\section{Within-trial analysis}

The within-trial analysis will investigate the cost-effectiveness of NIM compared with CM through a number of different analyses. The primary within-trial analysis will be a cost-utility analysis (CUA) which will estimate the incremental cost per quality-adjusted life year (QALY) of NIM compared with CM. QALYs will be generated via measurement of utility values using the Paediatric Quality of Life Inventory (PedsQL) ${ }^{14}$ child health-related quality of life (HRQoL) instrument mapped to the EQ-5D (ie, a widely used instrument to assess HRQoL) to generate utility values. ${ }^{15}$ Furthermore, the same authors who mapped the PedsQL to the EQ-5D are currently developing a preference-based index for the PedsQL. As this is ongoing research, the inclusion of this preference-based index will be explored at time of analysis, if available. This mapping of the PedsQL to QALY represents a strength of this project, since it will allow the estimation of child-specific health utilities. ${ }^{15}$ Additionally, the incremental cost per unit improvement using the effectiveness outcome $\mathrm{SDQ}^{16}$ will be explored. Within such a technical efficiency framework, there is no accepted threshold value for unit changes in the SDQ however, the costs required to reduce total difficulties scores (and remove altogether) will be reported.

Increasingly, in complex public health evaluation research, there are questions about whether all relevant benefits can be captured in a single summary outcome measure such as QALY or unit of 'effectiveness' or net benefit approach ${ }^{17}$ which is why the use of a cost-consequence analysis (CCA) framework is being recommended for such economic evaluations. ${ }^{18}$ Other outcomes from the trial such as the Parent-Infant Relationship Global Assessment Scale (an observational measure that is independently rated blind to group allocation) will be included in the CCA so that all costs and outcomes from the trial can be displayed transparently for decision-makers to consider trade-offs themselves. This format can be particularly useful in capturing broader intervention effects not contained within the psychometric properties of the PedsQL or SDQ.

These analyses will adhere to good practice guidelines for conducting economic evaluations alongside clinical trials, reporting standards and the most recent National Institute for Health and Care Excellence (NICE) public health reference case. ${ }^{18-20}$ The within-trial analysis will adopt the perspective of the NHS and Personal Social Services (PSS) and examine the costs to these sectors specifically. Additionally, a wider public sector perspective will be explored which will include societal resources such as contacts with the police, residential or respite care, and costs to the family in terms of additional child care.

\section{Resource use}

\section{Identification of resource use}

The identification of the resources used within such a complex intervention relates to the identification of multiple components involved in the delivery of NIM and $\mathrm{CM}$ interventions, as well as identification of the costs incurred and cost savings arising as a consequence of the intervention and the control.

The costs borne by the health and social care (NHS/ PSS) to deliver the NIM and the CM interventions include the time spent by individuals delivering the NIM and CM services, such as medical professionals and service management (administrators, team leaders, team members, area social workers, psychologists and psychiatrists). In addition, the consequential health and social services used by participants (mental health services, 
admissions to hospital, addiction/domestic violence services etc) will be also taken into account.

The time spent by birth parents and other primary caregivers' involvement in NIM or CM as well as police contacts, day care, school or nursery usage will be incorporated into the calculation of scenario analyses to provide a broader societal perspective of the costs of these services.

\section{Measurement of resource use}

Data on the time spent by various practitioners (eg, social workers, administrators, psychologists etc) in providing each service will be measured from the services directly. Both NIM and CM collect information about each contact the service has with each child. This information includes the purpose, place, length of contacts and all individuals present at the meeting. The services also collect information about other services that the child and their birth parents or other primary caregivers were recommended to attend (eg, addictions support, women's support etc).

\section{New Orleans intervention model}

Total costs for NIM will comprise the cost of delivering NIM plus resources used during the trial period. To determine the cost of the intervention an approximate care pathway was defined with the help of key social care and infant mental health experts. This includes: (1) a standardised assessment taking approximately 12 weeks; (2) treatment which could consist of up to six different types of intervention; (3) post-treatment meetings; (4) debriefing and (5) any other substantial activity such as a court hearing or court attendance. Data on the use of this service are collected by the NSPCC's data collection system. This system routinely collects data on each contact for each child including the practitioner providing the service, all the individuals who were in attendance and the duration of the contact. This system is reliant on the routine input from NSPCC staff members and also collects information about other services that were recommended by practitioners for the birth parents/other primary caregivers. An example of the data that is extracted is given in online supplementary appendix 1 .

The NSPCCs data collection system does not collect the key resource use items of preparation and administrative staff time for each contact with or for the child in question. An informal Delphi technique will be used to estimate the likely average, minimum, and maximums of the preparation and administrative time that goes into each contact, precontact and postcontact. NIM team members in Glasgow and London will be surveyed individually to provide their estimates, and those estimates will be combined to provide averages with uncertainty estimates on either side. The team will then be consulted again with these estimates to come to a final consensus.

\section{Case management}

CM provides social work service as usual, enhanced through the standardisation that comes with the RCT. This includes regular multiagency meetings that troubleshoot problems with services and individual cases. Information on date, the nature of the contact, duration, attendees, and if they were referred to an outside service are all collected electronically and held on a shared drive as multiple people may work on existing cases. Duration includes time spent before (preparing and setting up the meeting), during and after (writing up notes, updating case files and report writing) the contact. In Glasgow, there are typically eight observed contacts and six individual interviews over approximately 4 months and the intensity of contacts remains to be seen in London. However, the case may remain open for some time after the initial contacts, for example, to attend court dates or other judicial meetings. Therefore, any further contacts for each case will be recorded at their 15-month and 2.5-year follow-ups. The form used in collecting resource use from contacts is provided in online supplementary appendix 2.

\section{Additional service use}

Additional service use (ASU) data will be collected with questionnaires at baseline and at each point of follow-up. The ASU questionnaire (see online supplementary appendix 3) aims to obtain an estimate about the usage of services beyond those provided directly by NIM and CM.

This questionnaire asks birth parents and other primary caregivers about the number of attendances, contacts and hours for several different services (eg, hospital admissions, police contacts, day care or nursery usage etc) for both the child and themselves. These additional services are important resource use items to capture as CM and NIM both sometime refer patients out to these additional services, so the ASU provides a method that attempts to capture these.

The ASU Questionnaires will be compared with service use at baseline and used in conjunction with data from the NIM and CM services about recommended service uptake to identify what services were used resulting from involvement in either the CM or NIM services.

\section{Valuation of resource use}

\section{Unit costs}

Unit costs for each component of resource use will be expressed in pounds sterling $(£)$ for a base cost year 2020/2021, unit costs will be obtained from routine sources (ie, NHS Agenda for Change Pay scales, the Personal Social Services Resource Unit, NHS reference costs) or will be collected from the trial directly where they are not available in routine sources. The Hospital and Community Health Services pay and price index ${ }^{21}$ will be used to for any inflation.

\section{Outcomes}

A number of different outcomes will be collected by the trial. ${ }^{12}$ The primary outcome measures of interest for the economic evaluation are the PedsQL ${ }^{14}$ and the $\mathrm{SDQ}^{16}$ scores, both of which will be used in the economic evaluation. 
The PedsQL questionnaire is used to measure HRQoL in children aged $2-18$. The PedsQL is a validated measure of child quality of life ${ }^{112223}$ which has recently been mapped to utility values for use in health economic evaluations. ${ }^{24}$ The PedsQL has demonstrated responsiveness, construct validity and predictive validity in paediatric patients. The PedsQL scores can be mapped to generic EQ-5D utilities, facilitating calculation of QALYs, ${ }^{15}$ thus meeting most recent NICE guidance for public health interventions. ${ }^{25}$

The SDQ is a short behavioural screening questionnaire that is completed by parents, guardians or teachers of children aged 2-16 which records any emotional and behavioural difficulties and any impairment ${ }^{26}$ experienced by the child or family. Children can self-complete from ages 11 to 17 . The SDQ is one of the most widely used validated measure of mental health in children and is sensitive to change; in intervention studies, effect sizes have been shown to be moderate to large. ${ }^{27-29}$

Tables 1 and 2 provide a summary list of the economic evaluation measures, their schedule collection and the framework for analysis each will be used in. As recruitment is rolling, there is no set time for baseline, 15-month and 2.5-year data collection.

\section{Analysis of cost and effects}

Regression analysis will explore the effect that baseline variables have on the cost and effect (PedsQL and utility) of each intervention over a 2.5-years' time horizon. The incremental difference in cost and QALYs between groups at follow-up will be assessed while adjusting for baseline characteristics such as site, language, gender, baseline PedsQL and deprivation. Appropriate methodologies will be used in order to deal with the potential clustering of costs and outcomes by household and intervention site (eg, multilevel models) and with non-normality and correlation of cost and outcome data.

Two incremental cost-effectiveness ratio (ICER) will be calculated to evaluate the incremental cost per QALY (calculated from PedsQL scores) and the incremental cost per improvement in SDQ.

The ICER formula is given below:

$$
\mathrm{ICER}=\frac{\text { Cost }_{\mathrm{NIM}}-\text { Cost }_{\mathrm{CM}}}{\text { Effect }_{\mathrm{NIM}}-\text { Effect }_{\mathrm{CM}}}
$$

A CCA will be presented in tabular format with costs collected from the resource used in the trial presented on one side (table 1) and all outcomes listed along the other side (table 2).

Subgroup analysis will explore heterogeneity of the cost-effectiveness results by age group and intervention site.

To reduce bias and increase statistical power, missing information for the key variables used in the BeST? trial will be imputed separately for each of the two arms of the trial using multiple imputation by chained equations. ${ }^{30-32}$

Costs and outcomes will be discounted at $1.5 \%$ as recommended for public health economic evaluations. ${ }^{18}$
Table 1 Economic evaluation resource use measures

$\begin{array}{lll}\begin{array}{l}\text { Resource use } \\ \text { category }\end{array} & \begin{array}{l}\text { Description of resource } \\ \text { used }\end{array} & \begin{array}{l}\text { Unit of } \\ \text { measure }\end{array}\end{array}$

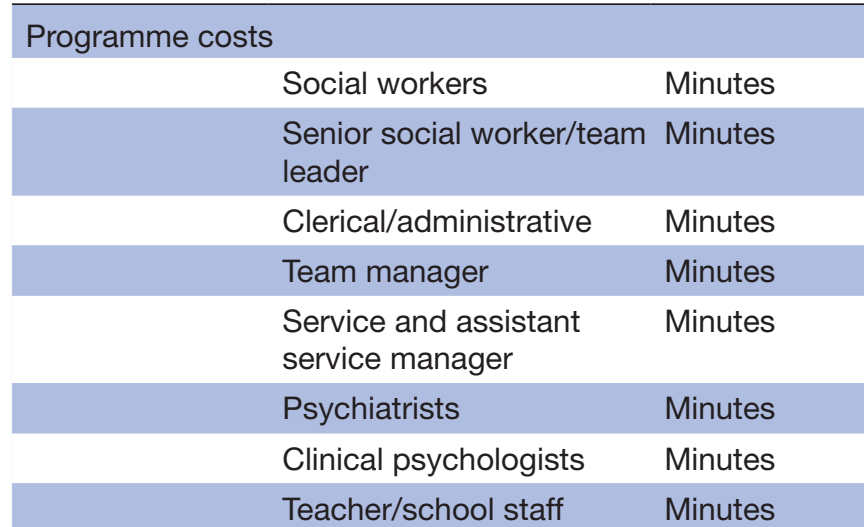

\section{Service use}

\begin{tabular}{|c|c|c|}
\hline & General practitioner & No. visits \\
\hline & Paediatrician & No. visits \\
\hline & Counsellor & No. visits \\
\hline & Health visitor & No. visits \\
\hline & Medical professional & No. visits \\
\hline & Psychotherapist & No visits \\
\hline & Dentist & No. visits \\
\hline & Hospital outpatient & No. visits \\
\hline & Hospital inpatient & No. visits \\
\hline & $\begin{array}{l}\text { Accident and emergency } \\
\text { visit }\end{array}$ & No. visits \\
\hline & Addiction services & No. visits \\
\hline & $\begin{array}{l}\text { Domestic violence } \\
\text { services }\end{array}$ & No. visits \\
\hline & $\begin{array}{l}\text { Adult metal health } \\
\text { services }\end{array}$ & No. visits \\
\hline & $\begin{array}{l}\text { Chile and adolescent } \\
\text { mental health services }\end{array}$ & No visits \\
\hline \multicolumn{3}{|l|}{ Other } \\
\hline & Legal & No. contacts \\
\hline & Child protection officer & No. contacts \\
\hline & Police & No. contacts \\
\hline & Family liaison worker & No. contacts \\
\hline & Nursery staff & No. contacts \\
\hline \multicolumn{3}{|l|}{ Family } \\
\hline & $\begin{array}{l}\text { Time away from work/ } \\
\text { usual activities }\end{array}$ & Hours \\
\hline
\end{tabular}

\section{Handling uncertainty}

The uncertainty surrounding the estimate of incremental costs, QALYs and ICERs will be investigated by use of a non-parametric bootstrap of the cost and effect pairs for 1000 iterations. ${ }^{33}$ This uncertainty will then be presented on the cost-effectiveness plane with a $95 \%$ CI of the bootstrapped ICER estimated. Results will be summarised using a cost-effectiveness acceptability 
Table 2 Economic evaluation outcome measures

\begin{tabular}{|c|c|c|c|c|c|c|}
\hline Measure & Baseline & $\begin{array}{l}15 \\
\text { months }\end{array}$ & $\begin{array}{l}2.5 \\
\text { years }\end{array}$ & Respondent & Measuring? & $\begin{array}{l}\text { Economic } \\
\text { evaluation } \\
\text { framework }\end{array}$ \\
\hline PedsQL & $x$ & $x$ & $x$ & Parent/carer & Quality of life & $\begin{array}{l}\text { CUA/CCA/long-term } \\
\text { (LT) model }\end{array}$ \\
\hline SDQ & $x$ & $x$ & $x$ & $\begin{array}{l}\text { Parent/carer } \\
\text { teacher }\end{array}$ & Mental health & CEA/CCA/LT model \\
\hline PIR-GAS & $x$ & $x$ & $x$ & Direct observation & Relationship functioning & CCA \\
\hline $\begin{array}{l}\text { Emotional Signalling } \\
\text { Scale }\end{array}$ & $x$ & $x$ & $x$ & Direct observation & $\begin{array}{l}\text { Degree which child signals } \\
\text { emotions to caregiver }\end{array}$ & CCA \\
\hline ITSEA* & $x$ & $x$ & & Parent/carer & Infant functioning & CCA \\
\hline WPPSI† & & & $x$ & Direct observation & Cognition & CCA \\
\hline DAWBA $\ddagger$ & $x$ & & $x$ & Parent/carer & Psychiatric diagnosis & $\mathrm{CCA}$ \\
\hline $\mathrm{DAl} / \mathrm{RPQ}$ & $x$ & $x$ & $x$ & Parent/carer & $\begin{array}{l}\text { Reactive attachment } \\
\text { disorder }\end{array}$ & CCA \\
\hline TIMB & $x$ & $x$ & $x$ & Parent/carer & Carer commitment & $\mathrm{CCA}$ \\
\hline $\begin{array}{l}\text { Time for permanent } \\
\text { placement decision }\end{array}$ & & & $x$ & Routine data & $\begin{array}{l}\text { Time from first care episode } \\
\text { to permanent placement }\end{array}$ & CCA \\
\hline $\begin{array}{l}\text { Linkage to routine data } \\
\text { (eg, crime, employment } \\
\text { mental health journey) }\end{array}$ & & & $\mathrm{X}$ & $\begin{array}{l}\text { Routine data if } \\
\text { available }\end{array}$ & $\begin{array}{l}\text { Contact with birth } \\
\text { parents, court hearings or } \\
\text { appearances, etc }\end{array}$ & LT model \\
\hline
\end{tabular}

*Infant-toddler social emotional assessment (only used with youngest infants in whom SDQ not validated).

†Full-scale IQ measure.

‡Development and well-being assessment gives psychiatric diagnoses.

CCA, cost-consequences analysis; CEA, cost-effectiveness analysis; CUA, cost-utility analysis; DAI/RPQ, Disturbances of Attachment Interview/Relationship Problems Questionnaire; DAWBA, Development and Wellbeing Assessment; ITSEA, Infant-Toddler Social and Emotional Assessment; PedsQL, Paediatric Quality of Life Inventory; PIR-GAS, Parent-Infant Relationship Global Assessment Scale; SDQ, Strengths and Difficulties Questionnaire; TIMB, This Is My Baby; WPPSI, Wechsler Preschool and Primary Scale of Intelligence.

curve to reflect the probability of NIM being cost-effective at various willingness-to-pay thresholds, including the $£ 20000$ to $£ 30000$ /QALY threshold. ${ }^{24}$

Heterogeneity will be explored and subgroup analyses undertaken, for example, based on study site, age groups and other relevant subgroups which may impact on cost-effectiveness. Scenario analyses will be conducted to examine the effect of the costs and consequences of the services to the immediate family of the child in care. To this end, ICERs will be recalculated including the costs and effects to the birth families and other primary caregivers (largely the time spent by these individuals during the period over which the service was provided).

\section{Long-term population health economic model}

While the within-trial economic evaluation assumes a relevant time horizon of 2.5 years, modelling the longterm cost-effectiveness of the NIM intervention will consider the wide spectrum of cross-sectoral impacts and costs to society of the intervention ${ }^{34} 35$ over the lifetime.

While several studies have evaluated interventions aiming at improving children's' mental health in terms of effectiveness ${ }^{36}$ and cost-effectiveness, ${ }^{37-39}$ evidence over a lifetime horizon is limited ${ }^{4041}$ and non-health costs and consequences are rarely considered. ${ }^{9}$

However, improvements in child mental health are likely to have broad societal (health and non-health related) and long-lasting impacts on the child, including reducing the risk of poor physical health, problems with substance abuse, suicide or other mental health risks, involvement in crime. ${ }^{1229394243}$

Furthermore, in line with the theory of 'Investing in Child Health', ${ }^{44}$ the social rate of return of more resource-consuming interventions directed to improve child mental health is likely to be potentially high, thus justifying the additional costs sustained by the decision-maker. ${ }^{8} 39$

The aforementioned multisector lifetime decision model will be based on the theory of 'Investing in Child Health $^{44}$ and will be adapted to model the long-term cost and outcomes from NIM and CM in four key sectors in society: education system, child welfare system, criminal justice, NHS and PSS as informed by this theory.

Online supplementary appendix 4 illustrates the economic logic model developed for this study which will inform the basic structure of the model.

Pending within-trial results, a Markov model (a mathematical model used to model randomly changing 
systems where it is assumed that future states depend only on the current state not on the events that occurred before it) will be used to predict the multisector lifetime cost-effectiveness. ${ }^{45}$ Several studies have used this framework to evaluate interventions directed towards the improvement of mental health in both adult ${ }^{4647}$ and infant population. ${ }^{38}$

The economic evaluation of NIM over a long-term horizon will use the trial data and outcome measures at 15-month and 2.5-year follow-up as predictors for parameter estimates in the lifetime model, and will be further supported by evidence from a systematic literature review and available record routine linkage data.

Considering the broad societal impacts and the multidisciplinary nature of many complex public health interventions affecting mental health, the model will calculate lifetime cost and sector-specific outcomes for each of the four key sectors (social care, informal care, production losses, crime and education), ${ }^{48}$ for the NIM intervention in comparison to $\mathrm{CM}$ including resource use; patient management and pathways; the cross-sectoral impact and implications of early intervention; quality of life; mortality and adverse events.

Specifically, routine data and a systematic literature review will inform specific parameters that will link early interventions on infant mental health-and related improvements in quality of life-with better health, educational and occupational outcomes, lower crime rates and cost savings for the health sector and the entire society.

\section{Sensitivity analysis}

Given that the assumptions about causal links from the trial into the future may not be valid over a long time frame (ie, over a child's lifetime), extensive sensitivity analyses will be conducted to explore the effects of adjusting the underlying model parameter estimates and assumptions. Probabilistic sensitivity analysis around the longerterm estimates of costs, effects and cost-effectiveness of the NIM intervention versus CM will be performed using a 1000 iteration Monte Carlo simulation. Further, an alternative discount rate of $3.5 \%$ will be applied to costs and effects in line with NICE guidelines. ${ }^{24}$ Scenario analyses will explore altering some of the underlying model assumptions.

\section{DISCUSSION}

The economic evaluation alongside the BeST? has been prospectively designed to identify, measure, and value key resource and outcome impacts arising from the NIM compared with CM. This is the first economic evaluation of its kind in the UK and the addition of a long-term economic model, multiple economic evaluation frameworks and public health economic evaluation methods guidance, should provide decision-makers with a comprehensive guide as to the likely cost-effectiveness of NIM. Given the complexity of the economic evaluation, key practical steps assisted the design including the development of a health economics logic model and key stakeholder engagement to identify the full range of ASU impacts arising from the intervention.

The BeST? is comparing complex interventions within a complex social care environment. NIM is a tailored and resource intense intervention with contextual factors influencing the bespoke nature; this will make the findings less generalisable to contexts outside of the UK. Equally, even though CM ensures adherence to standardised procedure, certain cases may be much more resource intense affecting cost-effectiveness.

The planned economic evaluation does have some limiting factors. There is potentially a risk of bias due to measurement challenges. Different services delivered in the intervention and in the control arm required different systems to collect resource use information. Furthermore, while the data collected within the pilot study have been carried over to the BeST?, the data collection instruments which have been used differ slightly between the pilot and the main trial. Additionally, the control intervention is not standardised between the Glasgow and the London site. However, we have planned for this and where possible, steps (eg, preliminary checks of the data collected; informal contacts with professionals in charge of data collection) will be taken to minimise this risk.

The base case CUA relies on a utility measure that will not be directly measured by the children themselves, rather they will be mapped from the non-preference-based PedsQL to preference-based EQ-5D for adults. While this has its limitations, at the time of trial design (2011), there was not a validated paediatric preference-based quality of life measure.

Additionally, the Child Health Utility 9D (CHU9D) was validated for use in children in 2012 for ages $7-11^{49}$ which is older than the targeted population of children. The very young children included in the study will also pose difficulties in terms of outcome measurement, because many of the paediatric outcomes included in the trial have not been validated for children under the age of 2 including the PedsQL although by the final trial assessment point, all children will have reached at least age 2.5 years. Despite these limitations, the recent mapping of the PedsQL to QALY does also represent a strength of current work, since it has allowed the estimation of children-specific health utilities. ${ }^{15}$ Further, the challenges mentioned above will be addressed through use of multiple economic evaluation frameworks, giving decision-makers transparent and comprehensive findings with which to improve resource allocation for this vulnerable population.

Given the broader and long-term consequences that inadequate responses to neglected and abused children has for the health sector and the society, investing in child health represents a key priority for the decision-maker. In this regard, the current study will provide evidence on the long-term value for money provided by a mental health individual-based intervention in the UK context. 


\section{Ethics and dissemination}

Results of the main trial and economic evaluation will be submitted for publication in a peer-reviewed journal as well as published in the peer-reviewed National Institute for Health Research journals library (Public Health Research Programme).

\section{Acknowledgements We thank the BeST? study team for their collaboration.}

Contributors MD drafted the economic evaluation protocol, KAB, NRSB and EM revised drafts. $K A B$ and $E M$ lead the design of the economic evaluation of the BeST? study. HM led the design of the BeST? study. EM, NRSB, HM, JD, KB and KAB commented on the final version.

Funding This work is supported by the National Institute for Health Research (public health research) grant number (12/211/54).

Competing interests None declared.

Patient consent Not required.

Ethics approval Ethics approval was obtained from the West of Scotland Ethics Committee 5.

Provenance and peer review Not commissioned; externally peer reviewed.

Open Access This is an Open Access article distributed in accordance with the Creative Commons Attribution Non Commercial (CC BY-NC 4.0) license, which permits others to distribute, remix, adapt, build upon this work non-commercially, and license their derivative works on different terms, provided the original work is properly cited and the use is non-commercial. See: http://creativecommons.org/ licenses/by-nc/4.0/

(C) Article author(s) (or their employer(s) unless otherwise stated in the text of the article) 2018. All rights reserved. No commercial use is permitted unless otherwise expressly granted.

\section{REFERENCES}

1. Fang X, Brown DS, Florence CS, et al. The economic burden of child maltreatment in the United States and implications for prevention. Child Abuse Negl 2012;36:156-65.

2. Corso PS, Edwards VJ, Fang X, et al. Health-related quality of life among adults who experienced maltreatment during childhood. Am J Public Health 2008;98:1094-100.

3. Knapp M, King D, Healey A, et al. Economic outcomes in adulthood and their associations with antisocial conduct, attention deficit and anxiety problems in childhood (In Submission). Journal of mental health policy and economics 2013;14:137-47.

4. Currie J, Widom CS, Spatz Widom C. Long-term consequences of child abuse and neglect on adult economic well-being. Child Maltreat 2010;15:111-20.

5. Olds D, Henderson, Jr CR, Cole R, et al. Long-term Effects of Nurse Home Visitation on Children's Criminal and Antisocial Behavior. JAMA 1998;280:1238-44.

6. Hannon C, Wood C, Bazalgette L. In Loco Parentis London: DEMOS, 2010.

7. Goldhaber-Fiebert JD, Snowden LR, Wulczyn F, et al. Economic evaluation research in the context of Child Welfare policy: a structured literature review and recommendations. Child Abuse Negl 2011;35:722-40.

8. Belli PC, Bustreo F, Preker A. Investing in children's health: what are the economic benefits? Bulletin of the World Health Organization 2005;83:777-84.

9. Beecham J. Annual research review: Child and adolescent mental health interventions: a review of progress in economic studies across different disorders. J Child Psychol Psychiatry 2014;55:714-32.

10. Zeanah CH, Larrieu JA, Heller SS, et al. Evaluation of a Preventive Intervention for Maltreated Infants and Toddlers in Foster Care. Journal of the American Academy of Child \& Adolescent Psychiatry 2001;40:214-21.

11. Boyd KA, Balogun MO, Minnis H. Development of a radical foster care intervention in Glasgow, Scotland. Health Promot Int 2016;31.

12. Minnis H, Boyd K, Fitzpatrick B, et al. Protocol 15PRT/6090:The Best Services Trial (BeST?): effectiveness and cost-effectiveness of the New Orleans Intervention Model for Infant Mental Health NCT02653716: The Lancet, 2016.

13. The good enough home? Home environment and outcomes of young maltreated children. Child \& Youth Care Forum, 2012. Springer.
14. Varni JW, Seid M, Rode CA. The PedsQL: measurement model for the pediatric quality of life inventory. Med Care 1999;37:126-39.

15. Khan KA, Petrou S, Rivero-Arias O, et al. Mapping EQ-5D utility scores from the PedsQL ${ }^{\mathrm{TM}}$ generic core scales. Pharmacoeconomics 2014;32:693-706.

16. Goodman R. The Strengths and Difficulties Questionnaire: a research note. J Child Psychol Psychiatry 1997;38:581-6.

17. Brazier J, Ratcliffe J, Salomon JA, et al. Measuring and Valuing Health Benefits for Economic Evaluation. 2nd edn. Oxford: Oxford University Press, 2017.

18. NICE. Methods for the development of NICE public health guidance (third edition, 2012. nice.org.uk/process/pmg4

19. Glick HA, Doshi JA, Sonnad SS, et al; Economic Evaluation in Clinical Trials. 2 ed. USA: Oxford University Press, 2015.

20. Husereau D, Drummond M, Petrou S, et al. Consolidated Health Economic Evaluation Reporting Standards (CHEERS) statement. Cost Eff Resour Alloc 2013;11:6.

21. Curtis L. Unit Costs of Health and Social Care. University of Kent: Personal Social Services Research Unit, 2014.

22. Everson-Hock ES, Jones R, Guillaume L, et al. The effectiveness of training and support for carers and other professionals on the physical and emotional health and well-being of looked-after children and young people: a systematic review. Child Care Health Dev 2012;38:162-74.

23. Craig P, Dieppe P, Macintyre S, et al; Developing and evaluating complex interventions: new guidance. In: Council MR, ed, 2008.

24. NICE, ed. Guide to the methods of technology appraisal 2013. Process and methods guides, 2013.

25. Petrou S, Gray A. Economic evaluation alongside randomised controlled trials: design, conduct, analysis, and reporting. BMJ 2011:342:d1548.

26. Palmieri PA, Smith GC. Examining the structural validity of the Strengths and Difficulties Questionnaire (SDQ) in a U.S. sample of custodial grandmothers. Psychol Assess 2007;19:189-98.

27. Tischler V, Vostanis $\mathrm{P}$, Bellerby $\mathrm{T}$, et al. Evaluation of a mental health outreach service for homeless families. Arch Dis Child 2002;86:158-63.

28. Gillberg C. The ESSENCE in child psychiatry: Early Symptomatic Syndromes Eliciting Neurodevelopmental Clinical Examinations. Res Dev Disabil 2010;31:1543-51.

29. Aoki $\mathrm{Y}, \mathrm{Zeanah} \mathrm{CH}$, Heller SS, et al. Parent-infant relationship global assessment scale: a study of its predictive validity. Psychiatry Clin Neurosci 2002;56:493-7.

30. Oostenbrink JB, Al MJ. The analysis of incomplete cost data due to dropout. Health Econ 2005;14:763-76.

31. Ramsey S, Willke R, Briggs A, et al. Good research practices for cost-effectiveness analysis alongside clinical trials: the ISPOR RCT-CEA Task Force report. Value Health 2005;8:521-33.

32. Ramsey SD, Willke RJ, Glick H, et al. Cost-effectiveness analysis alongside clinical trials II-An ISPOR Good Research Practices Task Force report. Value Health 2015;18:161-72.

33. Briggs $\mathrm{AH}$. Handling uncertainty in cost-effectiveness models. Pharmacoeconomics 2000;17:479-500.

34. Cleveland G, Krashinsky M. The Benefits and Costs of Good Child Care: The Economic Rationale for Public Investment in Young Children - A Policy Study: University of Toronto at Scarborough, 1998.

35. Heckman JJ. Return on Investment: Cost vs Benefits, 2008.

36. Marryat L, Thompson L, Wilson P. No evidence of whole population mental health impact of the Triple P parenting programme: findings from a routine dataset. BMC Pediatr 2017;17:40.

37. Duncan KM, MacGillivray S, Renfrew MJ. Costs and savings of parenting interventions: results of a systematic review. Child Care Health Dev 2017;43:797-811.

38. Bonin EM, Stevens M, Beecham J, et al. Costs and longerterm savings of parenting programmes for the prevention of persistent conduct disorder: a modelling study. BMC Public Health 2011;11:803.

39. Zerbe RO, Plotnick RD, Kessler RC, et al. Benefits and costs of intensive foster care services: The Casey Family programs compared to state services. Contemp Econ Policy 2009;27:308-20.

40. Stevens M. The cost-effectiveness of UK parenting programmes for preventing children's behaviour problems - a review of the evidence. Child Fam Soc Work 2014;19:109-18.

41. Kilian R, Losert C, Park A-L, et al. Cost-effectiveness analysis in child and adolescent mental health problems: an updated review of literature. Int J Ment Health Promot 2010;12:45-57. 
42. Smyke AT, Koga SF, Johnson DE, et al. The caregiving context in institution-reared and family-reared infants and toddlers in Romania. J Child Psychol Psychiatry 2007;48:210-8.

43. Knapp M, King D, Healey A, et al. Economic outcomes in adulthood and their associations with antisocial conduct, attention deficit and anxiety problems in childhood. $J$ Ment Health Policy Econ 2011:14:137-47.

44. Belli PC, Bustreo F, Preker A. Investing in children's health: what are the economic benefits? Bull World Health Organ 2005;83:777-84.

45. McDaid D. Economic modelling for global mental health. Global Mental Health Trials, 2014:265.

46. Siskind D, Araya R, Kim J. Cost-effectiveness of improved primary care treatment of depression in women in Chile. Br J Psychiatry 2010;197:291-6.
47. Siskind D, Baingana F, Kim J. Cost-effectiveness of group psychotherapy for depression in Uganda. $J$ Ment Health Policy Econ 2008;11:127-33.

48. Shearer J, McCrone P, Romeo R. Economic Evaluation of Mental Health Interventions: A Guide to Costing Approaches. Pharmacoeconomics 2016;34:651-64.

49. Stevens K. Valuation of the Child Health Utility 9D Index. Pharmacoeconomics 2012;30:729-47.

50. Bakermans-Kranenburg MJ, van IJzendoorn MH, Juffer F. Less is more: meta-analyses of sensitivity and attachment interventions in early childhood. Psychol Bull 2003;129:195-215.

51. Zeanah $\mathrm{CH}$, Larrieu JA, Heller SS, et al. Evaluation of a preventive intervention for maltreated infants and toddlers in foster care. J Am Acad Child Adolesc Psychiatry 2001;40:214-21. 